\title{
LIPOSOME-MEDIATED GENE TRANSFER IN RAT LUNG TRANSPLANTATION: A COMPARISON BETWEEN THE IN VIVO AND EX VIVO APPROACHES
}

Carlos H. R. Boasquevisque, MD ${ }^{a *}$

Bassem N. Mora, MD ${ }^{a}$

Mariano Boglione, $\mathrm{MD}^{\mathrm{a}}$

Jon K. Ritter, MD ${ }^{\mathrm{b}}$

Ronald K. Scheule, $\mathrm{PhD}^{\mathrm{c}}$

Nelson Yew, $\mathrm{PhD}^{\mathrm{c}}$

Lisa Debruyne, $\mathrm{MD}^{\mathrm{d}}$

Lihui Qin, MD ${ }^{\mathrm{d}}$

Jonathan S. Bromberg, MD ${ }^{\mathrm{d}}$

G. Alexander Patterson, MD ${ }^{\mathrm{a}}$
Objective: We compared the efficacy of in vivo and ex vivo liposome transfection in rat lung transplantation. Methods: (1) Chloramphenicol acetyltransferase group: Fischer rats underwent isogeneic transplantation ( $n=4$ per group). Recipients were put to death on postoperative day 2 for chloramphenicol acetyltransferase activity. $E x$ vivo setting: Grafts received cDNA complexed or not with liposomes and were transplanted after 1.5 or 10 hours at $10^{\circ} \mathrm{C}$. In vivo setting: Donors were intravenously injected with cDNA complexed or not with liposomes. Lungs were harvested after 1.5 or 10 hours, preserved at $10^{\circ} \mathrm{C}$, and transplanted. (2) Transforming growth factor- $\beta 1$ group: Brown-Norway rats served as donors and Fischer rats as recipients. All grafts were preserved for 3 hours at $10^{\circ} \mathrm{C}$. On postoperative day 5, arterial oxygenation and histologic rejection scores were assessed. Ex vivo setting: Grafts received transforming growth factor- $\beta 1$ sense $(n=8)$ or antisense $(n=7)$ complexed with liposomes or cDNA alone $(n=5)$. In vivo setting: Donors were intravenously injected with liposome:transforming growth factor- $\beta 1$ sense cDNA $(n=7)$. Exposure time was 3 hours. Results: (1) Chloramphenicol acetyltransferase-transfection was superior in the ex vivo group but was not statistically different for longer exposure times. (2) Transforming growth factor- $\beta 1$-arterial oxygenation was superior in the ex vivo liposome:sense group. cDNA alone was inefficient. Rejection scores were not statistically different between ex vivo and in vivo liposome:sense groups but were better when the ex vivo liposome:sense group was compared with the cDNA alone or the antisense groups. Conclusions: (1) With current liposome technology, the ex vivo route is superior to the in vivo approach; (2) cDNA alone does not provide transgene expression at levels to produce a functional effect. (J Thorac Cardiovasc Surg 1999;117:8-15)
W e have recently studied the feasibility, safety, and pattern of liposome-mediated gene transfer in a rat lung transplant model. ${ }^{1,2}$ We demonstrated that gene transfer to rat lungs can be achieved with minimal toxicity when grafts are transfected either before retrieval (in vivo) or after organs had been harvested (ex vivo). We also showed that gene therapy techniques can bring functional benefits to lung grafts. We observed that rat

From the Division of Cardiothoracic Surgery, ${ }^{a}$ Department of Surgery, and Department of Pathology, ${ }^{\mathrm{b}}$ Washington University School of Medicine, St Louis, Mo; Genzyme Corporation, ${ }^{\mathrm{c}}$ Framingham, Mass; and Department of Surgery, ${ }^{\mathrm{d}}$ University of Michigan, Ann Arbor, Mich.

Supported by the National Institutes of Health grants 1 R01 HL41281 and 1 F32HL09751-01.

Read at the Seventy-eighth Annual Meeting of The American Association for Thoracic Surgery, Boston, Mass, May 3-6, 1998. lung allografts transfected ex vivo with the immunosuppressive cytokine transforming growth factor- $\beta 1$ (TGF-ß1), complexed to liposomes, clearly showed improved functional results when compared with nontransfected allografts or allografts treated with TGF- $\beta 1$ antisense (unpublished data). These observations confirm the results of Qin, Ding, and Bromberg ${ }^{3}$ in the setting of experimental heart transplantation.

Received for publication May 8, 1998; revisions requested June 15, 1998; revisions received Aug 4, 1998; accepted for publication Sept 1, 1998.

Address for reprints: G. Alexander Patterson, MD, 3108 Queeny Tower, One Barnes-Jewish Hospital Plaza, St Louis, MO 63110.

*Supported by the Federal University of Rio de Janeiro-University Hospital Clementino Fraga, Filho, Brazil.

Copyright $\odot 1999$ by Mosby, Inc.

$0022-5223 / 99 \$ 8.00+0 \quad \mathbf{1 2 / 6 / 9 4 2 2 3}$ 
In the current study, we compared the in vivo and ex vivo approach in the setting of experimental lung transplantation, using a liposomal vector delivered via the vascular route. First, comparison was made using the reporter gene chloramphenicol acetyltransferase (CAT). Inasmuch as transgene expression per se does not assure a functional effect, the observations gathered in the CAT group were tested with the use of the functional gene TGF- $\beta 1$. Thus comparison between the ex vivo and in vivo approaches was also made on a functional basis.

\section{Materials and methods}

Plasmid expression vectors. The plasmid pCF1-CAT (Genzyme Corporation, Framingham, Mass) consists of the human cytomegalovirus immediate early gene promoter/ enhancer, a hybrid intron, the CAT cDNA, the bovine growth hormone polyadenylation signal sequence, and a kanamycin resistance gene, as previously described. ${ }^{4}$ The plasmids pMP6ATGF- $\beta 1$ sense and antisense were supplied by one of us (J. S. Bromberg, University of Michigan, Ann Arbor, Mich).

The pMP6ATGF- $\beta 1$ sense plasmid encodes for murine TGF- $\beta 1$ driven by the cytomegalovirus promoter. The pMP6ATGF- $\beta 1$ antisense plasmid contains the same sequence as the pMP6ATGF- $\beta 1$ sense plasmid except that the TGF- $\beta 1$ gene is inserted in an opposite direction in the plasmid, thus encoding no functional TGF- $\beta 1$ protein.

Liposomal vector. Lipid 67 (Genzyme Corporation) is an amphiphile consisting of a hydrophobic lipid anchor (cholesterol) linked to a spermine head group in a "T-shaped" configuration. Lipid 67:dioleoylphosphatidylethanolamine (DOPE), in a molar ratio of 1:2, was supplied as dried films and prepared as previously described. ${ }^{3}$ Liposome:cDNA complexes were prepared as described elsewhere. ${ }^{4}$

Animals. Inbred male Fischer and Brown-Norway rats weighing 270 to $300 \mathrm{~g}$ (Charles River Laboratories, Wilmington, Mass) were used in this study. Animals received humane care in compliance with the "Principles of Laboratory Animal Care" formulated by the National Society for Medical Research and the "Guide for the Care and Use of Laboratory Animals" prepared by the Institute of Laboratory Animal Resources and published by the National Institutes of Health (NIH Publication No. 85-23, revised 1985).

Rat lung transplantation. An orthotopic rat left lung transplant model was developed with the use of a modification $^{5}$ of the "cuff" technique. ${ }^{6}$ In brief, donor lungs were flushed through the main pulmonary artery with $20 \mathrm{~mL}$ of normal saline solution at a pressure of $10^{\circ} \mathrm{C}$ and $20 \mathrm{~cm} \mathrm{H}_{2} \mathrm{O}$. The heart-lung block was excised with the lungs inflated at end-tidal volume and the left pulmonary vein was cannulated with a silicone catheter inserted through the left atrial appendage. In the ex vivo setting, lipid 67:DOPE:cDNA was diluted in $5 \mathrm{~mL}$ of normal saline solution and infused retrogradely over 7 to 10 minutes at a pressure of $20 \mathrm{~cm} \mathrm{H}_{2} \mathrm{O}$. In the in vivo setting, grafts were infused with normal saline solution alone. Subsequently, the left lung was dissected and 14-gauge cuffs were inserted into the left pulmonary artery and vein. Grafts were implanted by means of the "cuff" technique for vascular anastomoses and suture technique for bronchial anastomoses (Prolene [Ethicon, Inc, Somerville, NJ] or nylon 8-0 or 9-0).

Experimental design. The study was divided into 2 main groups: CAT, which is a reporter gene, and TGF- $\beta 1$, which is a functional gene. Each group was subdivided into ex vivo and in vivo settings.

CAT group. Fischer rats underwent isogeneic left lung transplantation and were divided into 6 groups $(n=4$ per group). The preservation temperature was $10^{\circ} \mathrm{C}$ in all groups. Recipients were put to death on postoperative day 2 for measurement of CAT activity by means of the CAT assay as described elsewhere. ${ }^{7}$ Relative densitometry of the chromatographs of the CAT assay was performed with the use of Scion Image, an adaptation of the National Institutes of Health Image program for Windows 95.

EX VIVO SETTING. In group I, grafts were infused ex vivo with $660 \mu \mathrm{g}$ of CAT cDNA complexed to liposomes and then transplanted immediately, with a resultant exposure time of approximately 1.5 hours. Group II was similar to group I except that the exposure time was 10 hours at $10^{\circ} \mathrm{C}$. In group III, grafts were infused ex vivo with naked CAT cDNA alone, not complexed to liposomes, and then transplanted after an exposure time of approximately 1.5 hours at $10^{\circ} \mathrm{C}$.

IN VIVO SETTING. In group IV, donors received an intravenous injection of $1320 \mu \mathrm{g}$ of CAT cDNA complexed to liposomes via a cutdown approach to the left external jugular vein. After an exposure time of 1.5 hours in the animal, left lungs were harvested, preserved for an additional 1.5 hours at $10^{\circ} \mathrm{C}$, and then implanted. Group V animals were similar to group IV except that the exposure time was 10 hours in the animal and the preservation time was an additional 10 hours at $10^{\circ} \mathrm{C}$. In group VI, donors were injected with $1320 \mu \mathrm{g}$ of naked CAT cDNA by means of a similar approach. The exposure time in the animal was 1.5 hours, and the preservation time was an additional 1.5 hours at $10^{\circ} \mathrm{C}$ before implantation.

$T G F-\beta 1$ group. Brown-Norway rats served as donors and Fischer rats as recipients. There were 4 groups.

EX VIVO SETTING. In group I ( $\mathrm{n}=7$ ), Brown-Norway left lungs were transfected ex vivo with $660 \mu \mathrm{g}$ of TGF- $\beta 1$ antisense cDNA complexed to liposomes, preserved for 3 hours at $10^{\circ} \mathrm{C}$, and then transplanted into Fischer rats. In group II (n $=8$ ), Brown-Norway left lungs were treated in the same way, but were transfected with TGF- $\beta 1$ sense cDNA complexed to liposomes. In group III $(n=5)$, Brown-Norway grafts were transfected with naked TGF- $\beta 1$ sense cDNA.

IN VIVO SETTING. In group IV $(\mathrm{n}=7)$, Brown-Norway rats received an intravenous injection of $1320 \mu \mathrm{g}$ of TGF- $\beta 1$ sense cDNA complexed to liposomes via the left external jugular vein. After 3 hours of exposure in the animals, the left lungs were harvested, preserved for an additional 3 hours at $10^{\circ} \mathrm{C}$, and then transplanted.

No immunosuppressive drugs were used in any of the groups. All recipients were put to death on postoperative day 5 , based on previous studies (unpublished data). Arterial oxy- 

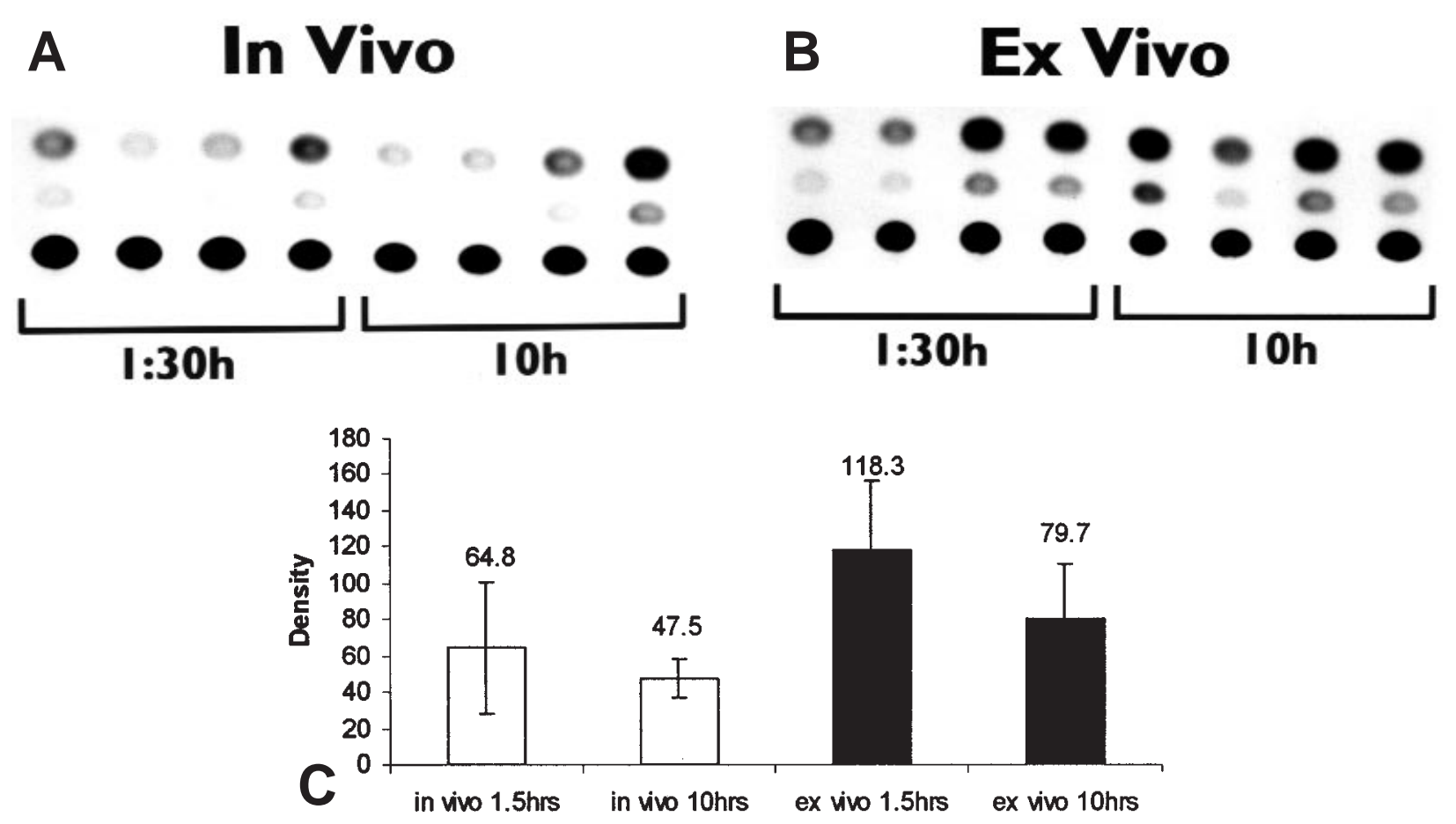

Fig 1. Lung CAT activity was analyzed by means of thin-layer chromatography. Each column corresponds to 1 animal. The bottom row represents non-acetylated chloramphenicol. The middle and top rows represent monoacetylated and diacetylated chloramphenicol, respectively. Overall, CAT activity was higher in the ex vivo (B) than in the in vivo setting (A); $P=.02$ (2-way analysis of variance; Fischer's least significant difference test). Interestingly, CAT activity was higher in grafts exposed for 1.5 hours than in grafts exposed for 10 hours, but the difference did not reach statistical significance: $P=.4$ for the in vivo setting and $P=.1$ for the ex vivo setting. $\mathbf{C}$, Relative densitometry of the chromatographs seen in A and B. Error bars represent standard deviations.

genation was measured after crossclamping of the right hilum, which resulted in the perfusion and ventilation of the transplanted left lung allograft only. An arterial blood sample was obtained from the aorta after left lung allograft ventilation for 5 minutes $(100$ strokes/min; inspired oxygen fraction $=1.0$; tidal volume $=1.5 \mathrm{~mL}$ ). After formalin fixation of the transplanted lungs, standard hematoxylin-eosin staining was performed. Histologic rejection was scored by a lung transplant pathologist blinded to the protocol according to the 1995 revision of the working formulation for the classification of pulmonary allograft rejection. ${ }^{8}$ Vascular and airway rejection scores were graded on a scale of 0 (no rejection) to 4 (complete graft destruction).

Statistical analysis. Data were expressed as mean \pm standard deviation. Data were compared by 1-way or 2-way analysis of variance and Fisher's post hoc multiple comparison technique as applicable, using the software Systat for Windows version 7 (Systat, Inc, Evanston, Ill, 1996).

\section{Results}

There were no operative deaths in any of the groups.

CAT group. CAT activity was superior in the ex vivo group, as demonstrated by the CAT assay chromato- grams. Increasing the exposure time from 1.5 to 10 hours did not improve transfection rate (Fig 1, $A, B$, and $C)$. Transfection with naked CAT cDNA resulted in transgene expression when infused by either the in vivo or ex vivo approaches.

TGF- $\beta 1$ group. Grossly, allografts treated ex vivo with liposome:TGF- $\beta 1$ sense cDNA were similar to native right lungs (Fig 3,C). Allografts treated in vivo showed a similar gross appearance. Conversely, lungs treated ex vivo with liposome:TGF- $\beta 1$ antisense cDNA or TGF- $\beta 1$ sense cDNA not complexed to liposomes showed diffuse hemorrhage and pulmonary edema (Fig $3, A$ and $B$ ).

Arterial oxygen tension was better in the ex vivo liposome:TGF- $\beta 1$ sense group than in the in vivo liposome:TGF- $\beta 1$ sense group and ex vivo liposome:TGF- $\beta 1$ antisense group. Allograft function was superior in both groups treated ex vivo and in vivo with liposome:TGF- $\beta 1$ sense compared with those treated with TGF- $\beta 1$ sense cDNA not complexed to liposomes (Fig 4).

Vascular rejection scores were $2.0 \pm 0.53(95 \% \mathrm{CI}$ $1.55-2.44)$ versus $2.35 \pm 0.47$ (95\% CI 1.91-2.79) ver- 


\section{cDNA versus cDNA + lipid}
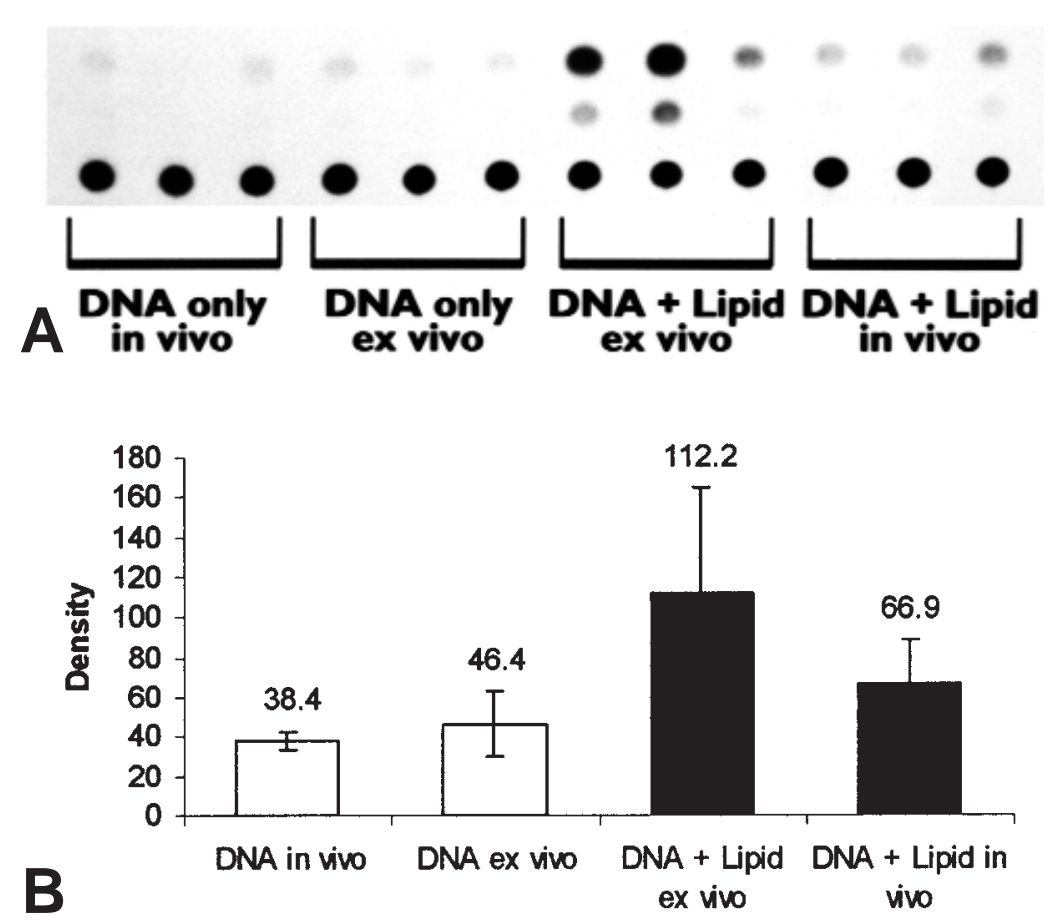

Fig 2. CAT activity in grafts treated with cDNA only versus cDNA complexed to liposomes. Each column corresponds to 1 animal. A, CAT activity was lower in lung isografts treated either in vivo or ex vivo with CAT cDNA alone in comparison with lung isografts treated in vivo or ex vivo with CAT cDNA complexed to liposomes. B, Relative densitometry of the chromatographs seen in A. Error bars represent standard deviations.

sus $2.83 \pm 0.68(95 \%$ CI $2.11-3.55)$ versus $2.6 \pm 0.22$ (95\% CI 2.32-2.87) for ex vivo liposome:TGF- $\beta 1$ sense, in vivo liposome-TGF- $\beta 1$ sense, ex vivo liposome:TGF- $\beta 1$ antisense, and ex vivo TGF- $\beta 1$ sense cDNA alone, respectively.

Airway rejection scores were $1.27 \pm 0.66(95 \% \mathrm{CI}$ $0.76-1.79)$ versus $1.57 \pm 0.34$ (95\% CI $1.25-1.89)$ versus $2.33 \pm 0.81(95 \%$ CI $1.47-3.19)$ versus $2.2 \pm 0.27$ (95\% CI 1.86-2.54) for ex vivo liposome:TGF- $\beta 1$ sense, in vivo liposome:TGF- $\beta 1$ sense, ex vivo liposome:TGF- $\beta 1$ antisense, and ex vivo TGF- $\beta 1$ sense cDNA alone, respectively.

There was no statistically significant difference between the ex vivo and in vivo liposome:TGF- $\beta 1$ sense groups $(P=.14$ and .33 for vascular and airway rejection scores, respectively; 1-way analysis of variance, Fisher's least significant difference test).

There was a statistically significant difference between the ex vivo liposome:TGF- $\beta 1$ sense and ex vivo liposome:TGF- $\beta 1$ antisense groups $(P=.009$ and .002 for vascular and airway rejection scores, respec- tively; 1-way analysis of variance, Fisher's least significant difference test).

There was no statistically significant difference in vascular rejection scores between the in vivo liposome:TGF- $\beta 1$ sense group and the ex vivo liposome:TGF- $\beta 1$ antisense group $(P=.19 ; 1$-way analysis of variance, Fisher's least significant difference test). There was, however, a statistically significant difference between these 2 groups when airway rejection scores were examined $(P=.03$; 1 -way analysis of variance, Fisher's least significant difference test). We did not observe any functional benefits in grafts treated ex vivo with TGF- $\beta 1$ sense cDNA alone, as measured by either allograft arterial oxygenation or rejection scores.

\section{Discussion}

In organ transplantation, the donated organ can be transfected either in vivo or ex vivo, that is, before or after retrieval. In lung transplantation, gene transfer can be achieved by delivering the vector:cDNA complexes via the pulmonary vasculature or the airways. 

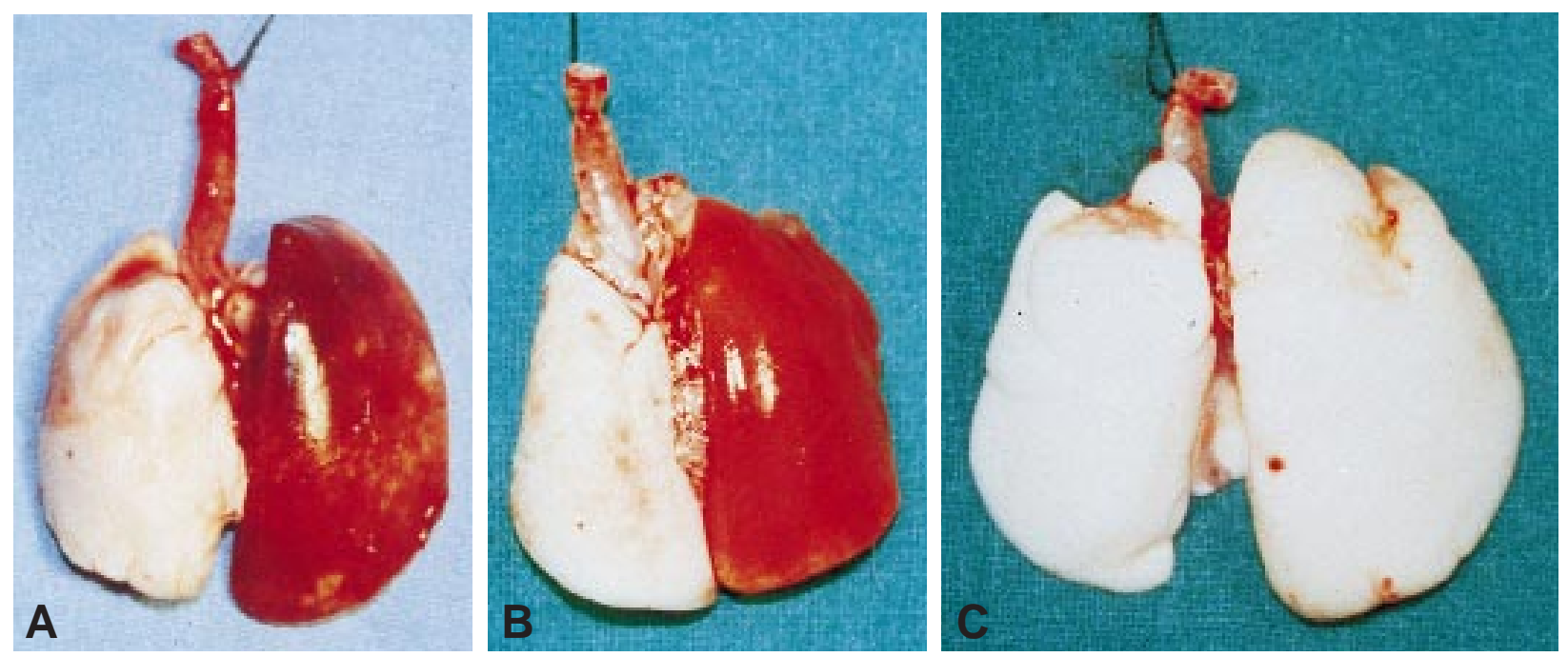

Fig 3. A, Gross pathology of a Brown-Norway allograft on posttransplantation day 5 treated ex vivo with liposome:TGF- $\beta 1$ antisense cDNA. Lungs showed extensive hemorrhage and edema, which was also observed in (B) allografts treated with naked TGF- $\beta 1$ sense cDNA. C, Gross pathology of a Brown-Norway allograft on posttransplantation day 5, treated ex vivo with liposome:TGF- $\beta 1$ sense cDNA. Allografts showed no edema or hemorrhage and were well ventilated.

We have recently described our results delivering the reporter gene CAT complexed to liposomes via the vascular route. ${ }^{1,2}$ When the liposome:cDNA complexes were delivered in vivo to the donor via an injection into the external jugular vein, transgene expression was strong in both hearts and lungs, minimal in livers, and not detectable in kidneys. ${ }^{1}$ Conversely, when lung grafts were infused ex vivo with liposome:cDNA complexes, transgene expression was minimal in hearts and not detected in livers and kidneys. The minimal transfection present in hearts could be eliminated by flushing the lung grafts with normal saline solution before implantation. Further, the duration of transgene expression was longer with the ex vivo approach than with the in vivo approach. ${ }^{1,2,9}$

In this current study, we compared the efficacy of both approaches using the vascular route. Our results demonstrate that the ex vivo strategy is more effective. In the CAT group, we observed superior transfection rates in the ex vivo liposome:CAT cDNA group than in the other groups. Importantly, this observation was affirmed with the use of TGF- $\beta 1$, whereby improvements in both graft function and histologic characteristics were observed after the ex vivo treatment of lung allografts with TGF- $\beta 1$ sense cDNA complexed to liposomes. Arterial oxygen tension 5 days after transplantation was superior in the ex vivo liposome:TGF$\beta 1$ sense group in comparison with the in vivo group or the ex vivo liposome:TGF- $\beta 1$ antisense group.
There was considerable variability in arterial oxygenation measurements in both the ex vivo and in vivo liposome:TGF $\beta 1$ sense groups. We also noticed variability of CAT activity within the same CAT groups, reflecting variable levels of gene transfer and production of the protein-of-interest in lungs treated under the same conditions. This could potentially explain the variable results observed in the TGF- $\beta 1$ group regarding arterial oxygen tension.

We believe that the amount of infused cDNA is similar between the in vivo and ex vivo groups for the following reasons. In the in vivo approach, most of the liposome:cDNA complexes injected intrajugularly remain in the heart and lungs, with minimal systemic transfection. ${ }^{1}$ Both the right and left lungs are affected when this approach is used. In the ex vivo approach, the entire liposome:cDNA complex enters the pulmonary vasculature, with resultant selective transfection of left lungs. Inasmuch as both lungs are transfected using the in vivo approach and only left lungs are transfected using the ex vivo approach, we doubled the dose of liposome:cDNA complexes for use in the in vivo approach. It should be stated, however, that increasing the amount of liposome:cDNA complexes delivered intravenously may improve the results in the in vivo setting. If this is true, it further strengthens the superiority of the ex vivo strategy in that lower amounts of liposome:cDNA complexes are used with lower systemic transfection rates. 


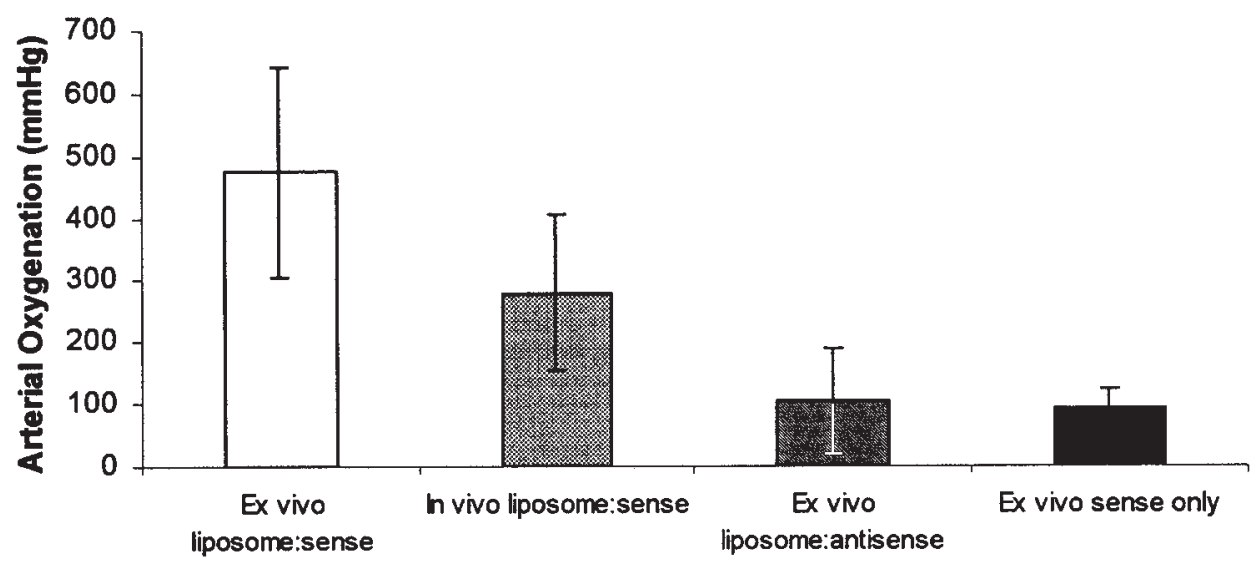

Fig 4. On posttransplantation day 5, allograft function was better in the ex vivo than in the in vivo liposome:TGF$\beta 1$ sense cDNA and TGF- $\beta 1$ sense cDNA alone groups $(474.2 \pm 168.5 \mathrm{~mm} \mathrm{Hg}$ [95\% CI 333.3-615.1] vs 279.9 $\pm 124.6 \mathrm{~mm} \mathrm{Hg}$ [95\% CI 164.6-395.2] vs $94.1 \pm 27.8 \mathrm{~mm} \mathrm{Hg}$ [95\% CI 59.5-128.7], respectively). In lungs transfected with liposome:TGF- $\beta 1$ antisense cDNA, arterial oxygen tension was $102.8 \pm 85.3 \mathrm{~mm} \mathrm{Hg}(95 \%$ CI 23.8 181.8). $P=.005$ for ex vivo versus in vivo liposome:sense; $P<.001$ for ex vivo liposome:sense versus ex vivo liposome:antisense or cDNA sense only; $P=.01$ for in vivo liposome:sense versus ex vivo liposome:antisense or cDNA sense only. Error bars represent standard deviations.

Histologic rejection scores in the ex vivo liposome:TGF- $\beta 1$ sense group did not differ significantly from those in the in vivo group, making it difficult to explain the better allograft function in the former. It should be stated, however, that histologic examination is a crude method to analyze the rejection process.

The results obtained in the ex vivo setting also demonstrate that ischemia and low temperatures do not interfere with the cellular uptake of cationic lipid:cDNA complexes, although they do delay transgene expression. We have previously demonstrated ${ }^{2}$ that transgene expression is not present during cold storage, even after prolonged incubation times of 12 hours at $10^{\circ} \mathrm{C}$, a temperature at which lungs are thought to still maintain aerobic metabolism. ${ }^{10}$

Transgene expression does occur in lung grafts when naked cDNA is administered without being complexed to liposomes. The amount of transgene expression, however, is at lower levels compared with cDNA complexed to liposomes, as was observed in the CAT group. However, the levels obtained are not high enough to produce a functional effect, as we demonstrated using the TGF- $\beta 1$ gene. Arterial oxygen tension and histologic rejection scores were similar to the ones observed in the control group transfected with liposome:TGF- $\beta 1$ antisense cDNA.

Finally, one should consider the several factors that may affect liposome-mediated gene transfer efficiency, such as (1) the neutral lipid used, (2) the structure of the cationic lipid moiety, (3) the size of liposomal particles (large multilamellar or small unilamellar vesicles), and
(4) host tissue factors. ${ }^{11}$ As we better understand the regulatory processes involved in DNA expression, we will be able to improve vector technology and enhance gene therapy strategies.

In summary, this study demonstrates that (1) with the use of current liposome technology, the ex vivo approach is superior to the in vivo approach in experimental lung transplantation when the liposome:cDNA complexes are delivered via the vascular route and (2) infusion via the pulmonary vasculature of naked cDNA that is not complexed to liposomes produces transgene expression, although not at levels sufficient to produce functional effects in this model.

We acknowledge the assistance of Richard B. Schuessler, $\mathrm{PhD}$, in the statistical analyses and Kathy Grapperhaus in the experimental assays.

\section{REFERENCES}

1. Boasquevisque CHR, Lee TC, Mora BN, et al. Liposome-mediated gene transfer to lung isografts. J Thorac Cardiovasc Surg 1997;114:783-92.

2. Boasquevisque CHR, Mora BN, Bernstein M, et al. Ex vivo liposome-mediated gene transfer to lung isografts. J Thorac Cardiovasc Surg 1998;115:38-44.

3. Qin L, Ding Y, Bromberg JS. Gene transfer of transforming growth factor- $\beta 1$ prolongs murine cardiac allograft survival by inhibiting cell-mediated immunity. Hum Gene Ther 1996;7: 1981-8.

4. Lee ER, Marshall J, Siegel CS, et al. Detailed analysis of structures and formulations of cationic lipids for efficient gene transfer to the lung. Hum Gene Ther 1996;7:1701-17.

5. Mizuta T, Kawaguchi AT, Nakahara K, Kawashima YI. 
Simplified rat lung transplantation using cuff technique. J Thorac Cardiovasc Surg 1989;97:578-81.

6. Shiraishi T, DeMeester SR, Worrall NK, et al. Inhibition of inducible nitric oxide synthase ameliorates rat lung allograft rejection. J Thorac Cardiovasc Surg 1995;110:1449-60.

7. Kitsis RN, Butrick PM, Kass AA, Kaplan ML, Leinwald LA. In: Adolph KW, editor. Methods in molecular genetics. New York: Academic Press; 1993. p. 374-92.

8. Yousem SA, Berry GJ, Cagle PT, et al. Revision of the 1990 working formulation for the classification of pulmonary allograft rejection: Lung Rejection Study Group. J Heart Lung Transplant 1996;15:1-5.

9. Mora BN, Boasquevisque CHR, Boglione M, et al. Stable longterm liposome-mediated gene expression is possible following repeat administration in lung transplant allografts [abstract]. Am J Respir Crit Care Med 1998;157:A333.

10. Date H, Matsumura A, Manchester JK, Cooper JM, Lowry OH, Cooper JD. Changes in alveolar oxygen and carbon dioxide concentration and oxygen consumption during lung preservation: the maintenance of aerobic metabolism during lung preservation. $\mathbf{J}$ Thorac Cardiovasc Surg 1993;105:492-501.

11. Liu Y, Mounkes LC, Liggit HD, et al. Factors influencing the efficiency of cationic liposome-mediated intravenous gene delivery. Nat Biotechnol 1996;15:167-73.

\section{Discussion}

Dr Hikaru Matsuda (Osaka, Japan). I congratulate you for your excellent results with a fine gene transfection model. In the group at Osaka University Medical School, we have been conducting gene transfection experiments using the HVJ liposome method. The target organs have been the liver, the heart, the kidneys, and recently the lungs. The efficacy has been very high compared with the conventional method including adenovirus vector and simple liposome method. Recently, using the HVJ method, my colleague, Dr Takeda, has shown an interesting result of producing the histologic changes similar to obliterative bronchiolitis seen in chronic lung transplant rejection using intratracheal injection of endothelin-1 gene. I agree that transplantation can provide a unique opportunity for gene transfer during harvest and storage in the ex vivo state to understand the mechanism of rejection, as well as to reduce the ischemic injury. How do you rate the efficiency of the liposome method in comparison with the other methods you have evaluated? Have you tried the intratracheal injection method?

Dr Boasquevisque. Thank you very much for your comments. Indeed, we are conducting some studies in which we are administering a liposomal vector complexed to TGF- $\beta 1$ cDNA through the airways. We are getting encouraging results.

Regarding the use of the HVJ-liposome vector, I think you are right. The ideal vector is not established yet, and much work has to be done in this field. It is worthwhile to try this vector.

Dr Thomas M. Egan (Chapel Hill, NC). I have 2 questions. Your outcome was gas exchange. Did you clamp the right hilum or did you just ventilate the left lung?

Dr Boasquevisque. During the assessment the right hilum was always clamped for 5 minutes
Dr Egan. Did you look at a product of gene expression and try to correlate that with rejection scores?

Dr Boasquevisque. We did try to measure the levels of TGF- $\beta 1$ using 2 different methods, luciferase and enzymelinked immunosorbent assay, but we did not succeed. We could not even show a difference between transfected and nontransfected normal lungs. That is why we used the CAT gene, with which it is easier to detect the protein expression.

Dr Walter Weder (Zurich, Switzerland). How much is known concerning the temperature dependence of transfection? Is there any transfection at $4^{\circ} \mathrm{C}$ and is $15^{\circ} \mathrm{C}$ better than $10^{\circ} \mathrm{C}$ ? Is there a difference in the temperature dependency between different vectors?

Dr Boasquevisque. We have conducted a series of experiments in which we checked the effect of temperature on transgene expression. First, we used the reporter gene CAT, and it seemed to have a better transfection rate at $10^{\circ} \mathrm{C}$. However, when we used the functional gene TGF- $\beta 1$, we did not detect any difference with regard to a functional benefit to the allograft, transfecting the lung at either $4^{\circ} \mathrm{C}$ or $10^{\circ} \mathrm{C}$.

With regard to the adenoviral vector, we have previously demonstrated that low temperatures such as $4^{\circ} \mathrm{C}$ or $10^{\circ} \mathrm{C}$ do not interfere with adenovirus-mediated gene transfer.

Mr Peter Goldstraw (London, England). I have a little difficulty with the equivalence of your dosages. You gave 660 $\mu \mathrm{g}$ into 1 lung and you doubled the dose when you put it into the whole body. Is that not just a dose effect?

Dr Boasquevisque. When we treated the donor in vivo, we injected the liposome:cDNA complexes through the jugular vein, so that the entire liposomal-DNA load would be directed to the heart-lung block. The problem is that part of the complex is trapped in the heart. With current liposome technology, there are anatomic barriers to overcome until the liposome-DNA load reaches the target, which is the lung in our study, when we use the in vivo approach. This does not occur in the ex vivo approach, in which the liposome:DNA complexes are delivered directly to the pulmonary vasculature. As a result, a much smaller amount is needed to transfect the lungs ex vivo. We probably could get the same results in the ex vivo approach using a smaller amount of liposome:DNA complex than we used in this current study, because we did not clamp the pulmonary artery while infusing the liposome:DNA complexes via the pulmonary vein. This fact per se strengthens the superiority of the ex vivo approach over the in vivo approach. Besides the advantage in terms of dosage, target specificity can be achieved with the ex vivo approach. Because of these facts, it seems to me that with current liposome technology, the ex vivo approach is more efficient than the in vivo approach when the vascular route is used.

Dr Larry R. Kaiser (Philadelphia, Pa). Do you have any indication of how long transgene expression persists with the ex vivo versus the in vivo approach?

Dr Boasquevisque. Yes, we have published a study in which we demonstrated that, in the ex vivo approach, transgene expression is very strong 28 days after transfection, whereas in the in vivo approach, transgene expression was almost zero at 
1 day. On checking further, we showed that the transgene is expressed for 45 days when the lung is transfected ex vivo.

Dr Kaiser. In these models that you observed, do you see transgene expression in other organs whether you have transduced them ex vivo or in vivo? Are you seeing expression in the liver, for instance?

Dr Boasquevisque. When the lungs are transfected in vivo, through the intrajugular route, a very strong transgene expression will be seen in the heart, equivalent to what we observe in the lungs. There is minimal expression in the liver, and we could not detect expression in the kidneys. Using the ex vivo approach, we detect minimal transgene expression in the heart, which can be eliminated by giving an additional flush to the lung graft immediately before implantation.

Dr Shaf Keshavjee (Toronto, Ontario, Canada). Following along the lines of Mr Goldstraw's comment, I think we need to develop many ways of optimizing transfection to the donor organ. You have been looking at the transvascular route. It needs to be emphasized that your findings as to the efficacy of ex vivo transfection reflect the route that you have chosen. The ex vivo route gets around the problem of dilution and the dose effect that was mentioned, as well as the effect of time of exposure to the pulmonary endothelium. If you are going to use the pulmonary endothelial route, the vascular route, you are going to need directed gene therapy, either viral or liposomes that are targeted. Your findings may not generalize to ex vivo being better by the transtracheal route. Have you done those experiments with TGF- $\beta$ by the transtracheal route?

Dr Boasquevisque. With current liposome technology and the vascular route, the ex vivo approach is more efficacious. In the current study, we used the vascular route. However, the airway route is being used in another study in our laboratory, and we are getting similar results.

The vascular route is very attractive in the setting of lung transplantation, chiefly if we consider that the endothelium is the first structure of the graft to contact the host inflammatory cells.

Dr Keshavjee. One other small technical question: Do you clamp the vessels to increase the contact time and/or pressure in the vessels?

Dr Boasquevisque. We did not clamp the vessels. The liposome:DNA complexes were infused under a pressure of $20 \mathrm{~cm} \mathrm{H}_{2} \mathrm{O}$ through the pulmonary vein over a period of 7 to 10 minutes. We did not clamp the pulmonary artery because we were afraid of injuring the endothelium due to the resultant high pressure.

Dr Keshavjee. I think that little bit of injury may work to your advantage in terms of increasing liposomal uptake in the endothelium. 